\title{
Incorporating Prior Knowledge in Observability-Based Path Planning for Ocean Sampling
}

\author{
Francis D. Lagor ${ }^{\mathrm{a}}$, Kayo Ide ${ }^{\mathrm{b}}$, Derek A. Paley ${ }^{\mathrm{c}, *}$ \\ ${ }^{a}$ Department of Aerospace Engineering, University of Maryland, College Park, MD 20742, USA \\ ${ }^{b}$ Department of Atmospheric and Oceanic Science, University of Maryland, College Park, MD 20742, USA \\ ${ }^{c}$ Department of Aerospace Engineering and the Institute for Systems Research, University of Maryland, College Park, MD 20742, USA
}

\begin{abstract}
Observability-based path planning of autonomous sampling platforms for flow estimation is a technique by which candidate trajectories are evaluated based on their ability to enhance the observability of underlying flow-field parameters. Until now, observabilitybased path planning has focused primarily on forward-in-time integration. We present a novel approach that makes use of the background error covariance at the current time to account properly for uncertainty of the underlying flow. The reduced Hessian of an optimal, linear data-assimilation strategy properly accounts for prior knowledge in the linear case and must be full rank to infer the initial state. The reduced Hessian represents an observability Gramian augmented with an inverse prior covariance. We extend this concept to the nonlinear case to yield a new criterion for scoring candidate trajectories: the empirical augmented unobservability index. Solving the differential covariance Riccati equation of the Kalman filter for deterministic dynamics also properly accounts for prior knowledge in the linear case, but at a later time. The solution to this equation reveals the important distinctions between observability-based, augmented observability-based, and anticipated covariance-based path planning. Path planning based on this unobservability index in the presence of prior information yields the desired behavior in numerical experiments of a guided Lagrangian sensor in a two-vortex flow pertinent to ocean sampling.
\end{abstract}

Keywords: observability, ocean sampling, path planning

\section{Introduction}

Ocean-observing systems provide essential information on the state of the ocean for use in oceanographic, atmospheric, and climatological modeling and forecasting. One such system is Argo, a continuously deployed, global array of drifting platforms [1]. These sensor systems will continue to increase in coverage and sampling capabilities as demand for ocean data increases. Incorporation of autonomous sampling platforms reduces oceanographic uncertainty through the determination of advantageous routes for measurement collection in response to uncertainty in estimates of a real-time environmental process [2]. The flow measurements of ocean sampling vehicles are often their Lagrangian data, i.e., measurements of the vehicle position under the influence of the flow. One such sampling platform is the underwater glider, which is a buoyancy-driven vehicle that alters its depth in a sinusoidal manner to induce flow over attached wings to make forward progress [3, 4]. Sensor platforms like gliders are minimally actuated to extend endurance; planning efficient, feasible, and informative routes is therefore essential.

Observability is the property of being able to infer the initial state of a system or underlying model parameters by observing the system output over a fixed time interval. Many researchers have planned informative routes by considering the path's observability or empirical observability, which is an approxima-

\footnotetext{
${ }^{*}$ Corresponding author

Email address: dpaley@umd.edu (Derek A. Paley)
}

tion to observability for nonlinear systems. Hinson et al. [5] analytically derive a trajectory that maximizes the observability of inertial position and heading for a self-propelled vehicle in a uniform flow. They pose an optimal-control problem to choose a path that minimizes the condition number of the observability Gramian for the linearized dynamics. Unfortunately, analytical solutions to the optimal-control problem only exist in specialized cases, due to the non-differentiability and non-convexity of the cost functional [5]. This problem may be addressed with grid-based optimization such as in the multivehicle sampling algorithm of DeVries et al. [6], if the application permits. Quenzer and Morgansen [7] also perform a finite-dimensional optimization over a discrete set of constant turning rates for an empirical observability-based controller in a multi-vehicle helming application.

Another method for performing finite-dimensional optimization of observability is to consider evaluation over a family of pre-determined candidate trajectories. The Adaptive Sampling and Prediction (ASAP) field experiment in Monterey Bay performed a similar optimization over a family of coordinated sampling patterns with respect to a sampling performance metric [8]. In addition to reducing computational cost, this method permits integration of observability optimization with other control policies that may have generated the candidate trajectories. In prior work, we generated candidate trajectories that steer a vehicle to separating boundaries of invariant regions in a twovortex flow field $[9,10]$.

Previous observability-based path-planning research has only 
been forward-looking. We propose to score trajectories using a new measure, the augmented unobservability index, to quantify how much each path increases the observability of estimated flow parameters given prior information in the form of a background error covariance matrix. Incorporating prior information in adaptive sampling has been accomplished by maximizing the anticipated reduction in error covariance. For example, Bishop et al. [11] consider an adaptive network design problem by optimizing the forecasted error covariance of an Ensemble Transform Kalman Filter over a finite set of possible network realizations. Davis et al. [12] also consider the forecasted covariance reduction in an objective analysis estimation technique to simulate routes for underwater gliders. Anticipated error covariance analysis is similar in the case of a linear deterministic model to augmented observability. However, we highlight the distinctions in Section 3. In the nonlinear case, the approaches differ because the anticipated reduction in covariance approach depends on the estimation scheme. We define empirical augmented observability independently of the estimator; it includes only the system dynamics, output equations, and the background error covariance.

Our technical approach first considers the variational data assimilation strategy 4D-Var with deterministic, linear dynamics and uncertain measurements. These dynamics correspond to a tangent-linear approximation of a nonlinear system, similar to the tangent-linear model used in the definition of empirical observability by Krener and Ide [13]. The optimal solution of this problem requires inversion of a matrix known as the reduced Hessian. Since we formulate the reduced Hessian in terms of linear observability with the addition of an inverse background error covariance, we refer to it as the augmented observability Gramian. The minimum-variance solution for a posterior filter covariance is given by the continuous-time Kalman filter, which provides a differential Riccati equation describing its evolution. We derive the analytical solution to this differential Riccati equation by connecting the inverse covariance of a Kalman filter to the augmented observability. We extend the concept of augmented observability to the nonlinear setting using an empirical observability Gramian and derive an upper bound on the associated empirical augmented unobservability index. We also show how empirical augmented observability connects to information theory by demonstrating that empirical observability (without prior information) is an approximation to the Fisher information matrix.

The contributions of this paper are: (1) an analytical solution to a continuous-time 4D-Var variational data assimilation problem in terms of the linear stochastic observability Gramian with an inverse background error covariance, which we refer to as augmented observability; (2) an analytical solution to the continuous-time Kalman filter Riccati equation for a linear timevarying system with deterministic dynamics and uncertain measurements in terms of the stochastic observability Gramian; and (3) an extension of augmented observability to nonlinear systems based on the empirical observability Gramian, yielding a novel method for scoring candidate trajectories, the empirical augmented unobservability index. These contributions are important because they provide a quantitative evaluation crite- rion for automatic selection of the candidate path that maximizes the anticipated observability given existing state uncertainty. The strategy of path planning with empirical augmented observability is illustrated for a single vehicle in a two-vortex flow pertinent to ocean sampling. This example demonstrates that augmenting observability with prior information improves sampling by changing the optimal path in an intuitive manner.

Section 2 reviews empirical observability for nonlinear systems, the two-vortex system, and model-predictive path planning based on observability. Section 3 solves a linear 4D-Var variational data assimilation problem, defines the augmented observability Gramian, and derives the optimal inverse posterior covariance for a continuous-time Kalman filter with deterministic dynamics. Section 4 extends augmented observability to the nonlinear setting and presents numerical experiments showing path planning using the empirical augmented unobservability index. Section 5 summarizes the paper and ongoing research. The appendix connects empirical augmented observability and Fisher information, for the interested reader.

\section{Observability-Based Path Planning in a Two-Vortex Flow}

\subsection{Linear and Empirical Observability}

Observability describes the ability to infer the initial state of a system by observing the output over a specified time interval. Consider the linear system

$$
\dot{x}(t)=A(t) x(t), \quad y(t)=C(t) x(t)
$$

with $x(t) \in \mathbb{R}^{n}, y(t) \in \mathbb{R}^{m}, A(t) \in \mathbb{R}^{n \times n}$, and $C(t) \in \mathbb{R}^{m \times n}$. Observability can be assessed through inspection of the linear observability Gramian [14]

$$
\mathcal{W}_{\mathrm{o}}\left(t_{0}, t\right)=\int_{t_{0}}^{t} \Phi\left(\tau, t_{0}\right)^{T} C^{T}(\tau) C(\tau) \Phi\left(\tau, t_{0}\right) d \tau
$$

where $\Phi\left(\tau, t_{0}\right)$ is the state transition matrix for the dynamics from time $t_{0}$ to $\tau$. By uniqueness of the state solution to (1), the state transition matrix has the property that $\Phi\left(\tau, t_{0}\right)^{-1}=\Phi\left(t_{0}, \tau\right)$. Assessing the rank of $\mathcal{W}_{\mathrm{o}}\left(t_{0}, t\right)$ is a boolean test to determine whether the system is observable on the time interval $\left[t_{0}, t\right]$ : if $W_{\mathrm{o}}\left(t_{0}, t\right)$ is full rank, then the system is observable.

Next, consider the nonlinear system

$$
\dot{x}(t)=f(t, x(t)), \quad y(t)=h(t, x(t))+v(t),
$$

where $f$ and $h$ are nonlinear functions and $v(t)$ is white Gaussian noise with covariance $R(t)$. The tangent-linear model for the dynamics (3) along a reference trajectory $x_{r}(t)$ with output $y_{r}(t)$ is given by the linear system

$$
\frac{d}{d t}(\delta x(t))=\left.\frac{\partial f}{\partial x}\right|_{x_{r}(t)} \delta x(t), \quad \delta y(t)=\left.\frac{\partial h}{\partial x}\right|_{x_{r}(t)} \delta x(t) .
$$

For an initial condition $x_{0}$, the solution to (4) for $\delta x\left(t_{0}\right)=x\left(t_{0}\right)-$ $x_{r}\left(t_{0}\right)$ yields the approximations $x_{r}(t)+\delta x(t) \approx x(t)$ and $y_{r}(t)+$ $\delta y(t) \approx y(t)$. The local observability Gramian for the nonlinear system (3) is defined to be the linear observability Gramian (2) 
for the tangent-linear approximation (4) with $C(\tau)=\partial h /\left.\partial x\right|_{x_{r}(\tau)}$ and $\Phi\left(\tau, t_{0}\right)$ as the state transition matrix for $\partial f /\left.\partial x\right|_{x_{r}(\tau)}$ [13].

The empirical observability Gramian [13] is an approximation of the linear observability Gramian (2) for the nonlinear system (3). Let $\phi\left(\cdot, t_{0}, x\left(t_{0}\right)\right)$ denote the state solution of (3) from $\left(t_{0}, x\left(t_{0}\right)\right), h^{ \pm j}$ the system output corresponding to perturbed initial condition $x\left(t_{0}\right)^{ \pm j}=x\left(t_{0}\right) \pm \epsilon e_{j}$, and $e_{j}$ the unit vector with one in the $j$ th entry and zeros elsewhere. Through $2 n$ simulations of the system output for closely perturbed initial conditions, an empirical observability Gramian may be constructed as

$$
\mathcal{W}_{\mathrm{eo}}\left(t_{0}, t, x\left(t_{0}\right)\right)=\int_{t_{0}}^{t} \Psi_{\mathrm{e}}\left(\tau, t_{0}, x\left(t_{0}\right)\right)^{T} R^{-1}(\tau) \Psi_{\mathrm{e}}\left(\tau, t_{0}, x\left(t_{0}\right)\right) d \tau,
$$

where $\Psi_{\mathrm{e}}$ is an $n \times n$ matrix with $j$ th column specified by

$$
\left[\Psi_{\mathrm{e}}\left(\tau, t_{0}, x\left(t_{0}\right)\right)\right]_{j}=\frac{h^{+j}\left(\tau, \phi\left(\tau, t_{0}, x\left(t_{0}\right)\right)\right)-h^{-j}\left(\tau, \phi\left(\tau, t_{0}, x\left(t_{0}\right)\right)\right)}{2 \epsilon} .
$$

Note that that $\Psi_{\mathrm{e}}$ is a central-difference approximation to $\partial h /$ $\partial x\left(t_{0}\right)$ and, in the limit $\epsilon \rightarrow 0$ with $R(\tau)=\mathbb{I}, \mathcal{W}_{\text {eo }}$ converges to the local observability Gramian $\mathcal{W}_{\mathrm{o}}$ for $(3)[15,13]$. Inclusion of the $R(\tau)^{-1}$ term accounts for the affect of measurement covariance on observability and matches its placement within the linear stochastic observability Gramian, defined in the next section; in both the linear and nonlinear cases, the additive measurement noise does not influence the system dynamics. $\left(R(\tau)^{-1}\right.$ was omitted in [13], where it was noted that for systems with measurement noise, the outputs can be scaled so that the noise has unit covariance.)

Similar to the linear observability Gramian $\mathcal{W}_{\mathrm{o}}\left(t_{0}, t\right)$, the empirical observability Gramian $\mathcal{W}_{\text {eo }}\left(t_{0}, t, x\left(t_{0}\right)\right)$ must be full rank for the system (3) to be observable on $\left[t_{0}, t\right]$. However, the initial state $x\left(t_{0}\right)$ is also included in the arguments, because this assessment only applies for the initial condition used. $\mathcal{W}_{\text {eo }}$ has also been applied to systems similar to (3) that include a prescribed control signal $u(t)$, i.e., $\dot{x}(t)=f(t, x(t), u(t))[15,9]$. The control $u(\cdot)$ is a time-varying input signal, prescribed over $\left[t_{0}, t\right]$, so $\mathcal{W}_{\text {eo }}$ should be denoted $\mathcal{W}_{\text {eo }}\left(t_{0}, t, x_{0}, u_{\left[t_{0}, t\right]}\right)^{1}$.

In addition to providing a rank test for observability, $\mathcal{W}_{\mathrm{eo}}$ also provides a means of measuring the degree of observability. Krener and Ide [13] define the unobservability index $v$ of $\mathcal{W}_{\text {eo }}$ to be the reciprocal of the smallest eigenvalue $\lambda_{\min }$ of $\mathcal{W}_{\text {eo }}$, i.e.,

$$
v\left(\mathcal{W}_{\mathrm{eo}}\right)=\frac{1}{\lambda_{\min }\left(\mathcal{W}_{\mathrm{eo}}\right)} .
$$

The unobservability index quantifies how difficult it is to infer the initial condition $x\left(t_{0}\right)$ from the system output over $\left[t_{0}, t\right]$ [13].

\subsection{The Two-Vortex System}

The two-vortex system is a potential-flow model of two interacting ocean eddies that is useful in studying idealized pathplanning of ocean vehicles, because it is analytically tractable

\footnotetext{
${ }^{1}$ We omit some arguments of $\mathcal{W}_{\mathrm{eo}}\left(t_{0}, t, x\left(t_{0}\right), u_{\left[t_{0}, t\right]}\right)$ and write instead $W_{\text {eo }}\left(t_{0}, t\right)$ or $\mathcal{W}_{\text {eo }}$ if the meaning is clear from context.
}
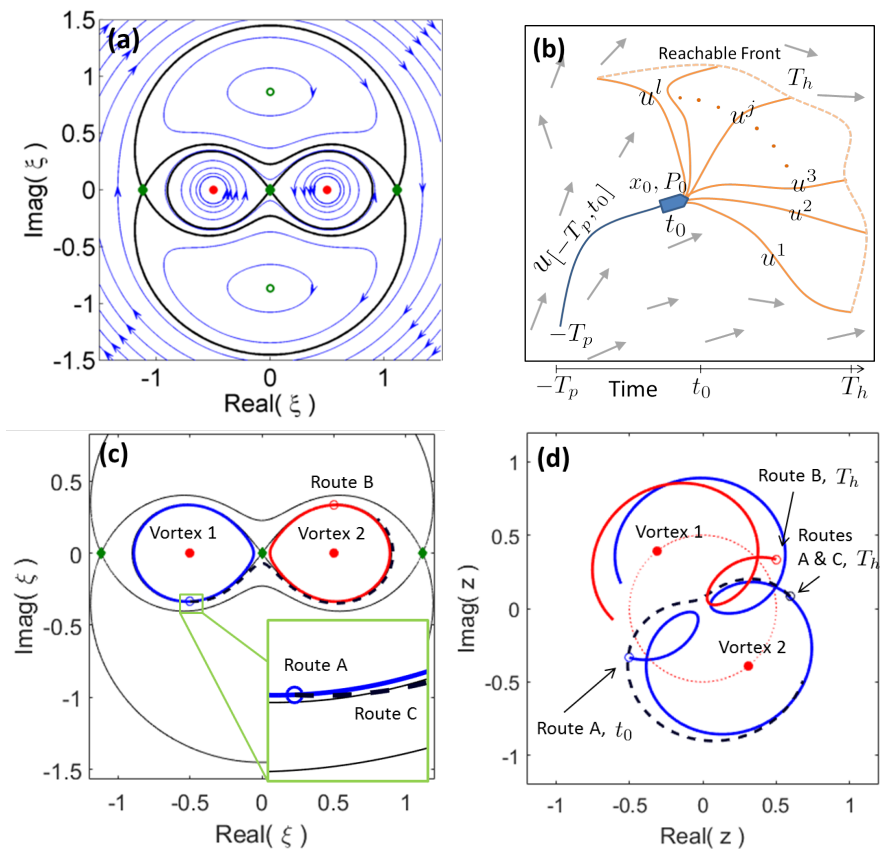

Figure 1: (a) Two-vortex system in the co-rotating frame; red circles are vortices; green circles and green diamonds are center and saddle fixed points, respectively; black lines are the stable and unstable manifolds of the saddle points. (b) Candidate trajectories in observability-based path-planning. (c) Routes in the observability example in the co-rotating frame. (d) Routes in the observability example in the inertial frame. Initial conditions for Route A: $X(0)=(1,-.5,0,1, .5,0,-.5,-.335)^{T}$; Route B: $X(0)=(1,-.5,0,1, .5,0, .5$, $.335)^{T}$; Route C: $X\left(T_{h}\right)=(1, .45,-.22,1,-.45, .22, .60, .081)^{T}$.

and (when viewed in a co-rotating frame) contains coherent flow structures [9]. These coherent structures are important barriers to transport that also play a role during path planning.

Let $z$ represent a location in the complex plane. The flow at location $z$ due to a vortex $j$ at $z_{j}$ with circulation strength $\gamma_{j}$ is

$$
f_{j}(z)=\frac{i \gamma_{j}}{2 \pi} \frac{z-z_{j}}{\left|z-z_{j}\right|^{2}}
$$

Vortices advect according to the flow of other vortices. Using (8), the equations of motion for two interacting vortices are $\dot{z}_{1}(t)=f_{2}\left(z_{1}(t)\right)$ and $\dot{z}_{2}(t)=f_{1}\left(z_{2}(t)\right)$. A kinematic model for an ocean vehicle located at $z$, advecting with the flow of two nearby vortices, and having control input $u(t) \in \mathbb{C}$ is

$$
\dot{z}(t)=f_{1}(z(t))+f_{2}(z(t))+u(t) .
$$

Two vortices rotate together in a relative equilibrium. The change of variables $z(t)=\xi(t) e^{i(\omega t+\theta)}$ with angular rate $\omega=\left(\gamma_{1}+\gamma_{2}\right) /$ $\left(2 \pi\left|z_{1}-z_{2}\right|^{2}\right)$ and initial phase angle $\theta$ transforms to a co-rotating frame described by the complex variable $\xi(t)$. In this frame, invariant regions appear as depicted in Fig. 1(a). The black lines in this figure represent separatrices, which divide invariant regions. A vehicle drifting within an invariant region may escape the region only if control actions are taken.

Although the above presentation of the two vortex system uses complex variables for compactness, the state of the twovortex system (including both vortices and a sampling vehicle) may be represented by the real-valued vector

$$
X=\left(\gamma_{1}, \operatorname{Re}\left(z_{1}\right), \operatorname{Im}\left(z_{1}\right), \gamma_{2}, \operatorname{Re}\left(z_{2}\right), \operatorname{Im}\left(z_{2}\right), \operatorname{Re}(z), \operatorname{Im}(z)\right)^{T} .
$$


Additionally, all empirical observability calculations in this paper for the two-vortex system are performed in the inertial frame and converted to the co-rotating frame only for plotting.

\subsection{Observability-Based Path Planning}

Observability-based path planning refers to the evaluation of a finite set of $l$ possible control signals $\left\{u^{j}(\cdot)\right\}_{j=1}^{l}$ over a forwardlooking time interval $\left[t_{0}, T_{h}\right]$ based on the anticipated observability of the output. (We do not consider generation of candidate control signals in this paper, only their evaluation.) Each control signal is scored using its unobservability index (7). The control signal that minimizes the unobservability index is selected, and the process may be repeated when re-planning is desired [10]. Figure 1(b) illustrates this process.

The primary contribution of this paper lies in the incorporation of prior information in observability-based path planning, motivated by the desire to evaluate forward-looking paths with consideration to where the vehicle has already been. The following example motivates incorporation of prior information.

Consider a drifting vehicle that has traversed Route A encircling Vortex 1 during time interval $\left[0, T_{h}\right]$, as shown in Fig. $1(\mathrm{c}) ; T_{h}$ is approximately 8.44 time units, corresponding to the time for the drifting vehicle to complete the blue orbit and return to its starting location in the co-rotating frame. After time $T_{h}$, the vehicle will again approach the saddle location at $\xi=0$. Fig. 1(c) shows two possible choices at $t=T_{h}$ : the vehicle can remain on Route A encircling Vortex 1, or switch to Route C, which requires a brief control action to cross the nearby separating boundary. (Route B will be addressed in Section 4.) After crossing, a vehicle traveling along Route $\mathrm{C}$ drifts to the right of the saddle at $\xi=0$ and partially encircles Vortex 2. Should the vehicle remain on Route A for the subsequent time interval $\left[T_{h}, 2 T_{h}\right]$ or exert a brief control effort to switch to Route C?

Table 1 shows the unobservability indices for the upper $6 \times 6$ submatrix of $\mathcal{W}_{\text {eo }}$ corresponding to the observability of the flow-field parameters and states $\gamma_{1}, \operatorname{Re}\left(z_{1}\right), \operatorname{Im}\left(z_{1}\right), \gamma_{2}, \operatorname{Re}\left(z_{2}\right)$, and $\operatorname{Im}\left(z_{2}\right)$, for these routes. Forward looking-observability on $\left[T_{h}, 2 T_{h}\right]$ shows Route A as preferable to Route C. However, if the previous empirical observability Gramian from $\left[0, T_{h}\right]$ for Route $\mathrm{A}$ is included in the evaluation, the route combination $(A, C)$ yields the most observable path. The value of the unobservability index over the interrupted intervals $\left[0, T_{h}\right],\left[T_{h}, 2 T_{h}\right]$ differs from the index over the continuous interval $\left[0,2 T_{h}\right]$, since new perturbed initial conditions are selected at time $T_{h}$ in the interrupted case. Moreover, note that the unobservability index for Route A differs between intervals $\left[0, T_{h}\right]$ and $\left[T_{h}, 2 T_{h}\right]$, because the vortices in the inertial frame are at a different phase of rotation for these times.

Table 1: Unobservability analysis of Routes A and C

\begin{tabular}{|c|c|c|c|c|c|}
\hline \multicolumn{2}{|c|}{ Time span: $\left[0, T_{h}\right]$} & \multicolumn{2}{|c|}{$\left[T_{h}, 2 T_{h}\right]$} & \multicolumn{2}{|c|}{$\left[0, T_{h}\right],\left[T_{h}, 2 T_{h}\right]$} \\
\hline Route & Unobs. & Route & Unobs. & Routes & Unobs. \\
\hline $\mathrm{A}$ & 6.222 & $\mathrm{~A}$ & 8.444 & $(\mathrm{~A}, \mathrm{~A})$ & 0.2927 \\
\hline $\mathrm{C}$ & - & $\mathrm{C}$ & 27.50 & $(\mathrm{~A}, \mathrm{C})$ & 0.2537 \\
\hline
\end{tabular}

Based on the results of this numerical experiment, including the observability for the portion of path already covered by a vehicle appears to be an appropriate method for incorporating historical information. However, the states of the vehicle and the flow field may be uncertain. Such evaluations should involve the best estimate of the system state at time $t_{0}$ and ideally incorporate the uncertainty about that state. In the sequel, we assume that all prior knowledge of the system up until time $t_{0}$ is encoded in a background error covariance matrix $P_{0}$.

\section{Augmented Observability}

\subsection{Error Covariance and Stochastic Observability}

Consider the time-varying, stochastic linear system

$$
\dot{x}(t)=A(t) x(t)+G(t) w(t), \quad y(t)=C(t) x(t)+v(t),
$$

where $w(t), v(t)$ are zero-mean stochastic processes with associated covariances $Q(t)$, and $R(t)$, respectively [16]. For simplicity, we suppress time-dependence in what follows.

The continuous-time Kalman filter provides the optimal, minimum-variance, unbaised estimate for the system (11). In addition, the Kalman filter provides an estimate of the error covariance, which describes the uncertainty in the state estimates. The error covariance $P(t) \in \mathbb{R}^{n \times n}$ evolves according to the differential Riccati equation [16]

$$
\dot{P}=A P+P A^{T}+G Q G^{T}-P C^{T} R^{-1} C P .
$$

Consider (11) with deterministic dynamics such that $Q(t) \equiv$ 0 . Then, (12) can be converted into an equivalent differential equation describing the evolution of $P^{-1}$ [16]

$$
\frac{d}{d t}\left(P^{-1}\right)=-P^{-1} A-A^{T} P^{-1}+C^{T} R^{-1} C
$$

with $P^{-1}\left(t_{0}\right)=0_{n \times n}$ for the case of no prior information. With the use of Liebniz's rule, the solution to this equation, verified via substitution, is [16]

$$
P^{-1}(t)=\int_{t_{0}}^{t} \Phi^{T}(\tau, t) C^{T} R^{-1} C \Phi(\tau, t) d \tau .
$$

The right-hand-side of (14) must be positive definite for some $t>t_{0}$ for observability [16]. This integral closely resembles the linear observability Gramian (2), except the arguments of $\Phi$ are $(\tau, t)$ instead of $\left(\tau, t_{0}\right)$. Multiplying (14) on the left by $\mathbb{I}=$ $\Phi^{T}\left(t, t_{0}\right)^{-1} \Phi^{T}\left(t, t_{0}\right)$ and on the right by $\mathbb{I}=\Phi\left(t, t_{0}\right) \Phi\left(t, t_{0}\right)^{-1}$, and using the composition property $\Phi\left(\tau, t_{0}\right)=\Phi(\tau, t) \Phi\left(t, t_{0}\right)$ yields

$$
\begin{aligned}
P^{-1}(t) & =\Phi^{T}\left(t_{0}, t\right)\left(\int_{t_{0}}^{t} \Phi^{T}\left(\tau, t_{0}\right) C^{T} R^{-1} C \Phi\left(\tau, t_{0}\right) d \tau\right) \Phi\left(t_{0}, t\right) \\
& =\Phi^{T}\left(t_{0}, t\right) W_{\mathrm{so}}\left(t_{0}, t\right) \Phi\left(t_{0}, t\right)
\end{aligned}
$$

where $\mathcal{W}_{\text {so }}$ defines the stochastic observability Gramian for (11) with $Q(t) \equiv 0$. $\mathcal{W}_{\text {so }}$ resembles the linear deterministic observability Gramian with an inclusion of $R^{-1}$ for measurement noise. Aoki [17] defined an analogous discrete-time stochastic observability Gramian containing the measurement noise covariance. The connection between error covariance and observability is apparent in the linear case: the inverse of the error covariance of the optimal filter for a linear time-varying system with deterministic dynamics is given by the stochastic observability Gramian transformed by $\Phi^{T}\left(t_{0}, t\right)$ and $\Phi\left(t_{0}, t\right)$. 


\subsection{D-Var solution to Optimal Linear Estimation}

4D-Var is a technique in data assimilation originating in numerical weather prediction and oceanography that provides smoothed estimates $^{2}$ of a trajectory of a dynamical system [18]. The name originates from the use of spatial, dynamical models of the weather and ocean (3D in space + 1D for time) as well as techniques from the calculus of variations. Under deterministic dynamics, solving for the optimal initial condition estimate $\hat{x}_{0}$ that results in a trajectory best fit to the data is equivalent to solving for the entire best-fit trajectory $\hat{x}(\cdot)$. Consider the following continuous-time, optimal estimation problem with a linear dynamical constraint. Given an estimate $\hat{x}_{0}$ of the initial state with covariance $P_{0}$, let $\hat{x}(\cdot)$ denote the nonlinear trajectory resulting from $\hat{x}_{0}$. Let $C$ denote the observation operator of the tangent-linear model (4), and let $\Delta y(t)=y(t)-\hat{y}(t)$ be the difference between measurement $y(t)$ and the expected measurement $\hat{y}(t)$ corresponding to estimate $\hat{x}_{0}$. We seek an updated estimate $\hat{x}_{0}+\delta x_{0}$ of the initial condition for which the update $\delta x_{0}$ minimizes (with $\tau$ arguments suppressed)

$$
J(\delta x)=\frac{1}{2} \int_{t_{0}}^{t}(\Delta y-C \delta x)^{T} R^{-1}(\Delta y-C \delta x) d \tau+\frac{1}{2} \delta x\left(t_{0}\right)^{T} P_{0}^{-1} \delta x\left(t_{0}\right)
$$

$$
\text { subject to } \frac{d}{d t}(\delta x(t))=\left.\frac{\partial f}{\partial x}\right|_{\hat{x}(t)} \delta x(t) \quad \text { with } \quad \delta x\left(t_{0}\right)=\delta x_{0} .
$$

The cost function (16) has continuous-time measurements but is otherwise identical to the incremental 4D-Var approach of Courtier et al. [19]; eqn. (16) can therefore be thought of as the incremental cost function in the inner loop of a nonlinear 4D-Var implementation. Since the dynamics are linear and deterministic, we may solve (17) in terms of $\delta x_{0}$, i.e.,

$$
\delta x(t)=\Phi\left(t, t_{0}\right) \delta x_{0} .
$$

Using (18) and $\delta x\left(t_{0}\right)=\delta x_{0}$ converts (16) to the unconstrained cost function

$$
\bar{J}\left(\delta x_{0}\right)=\frac{1}{2} \int_{t_{0}}^{t}\left(\Delta y-C \Phi \delta x_{0}\right)^{T} R^{-1}\left(\Delta y-C \Phi \delta x_{0}\right) d \tau+\frac{1}{2} \delta x_{0}^{T} P_{0}^{-1} \delta x_{0} .
$$

A first-order necessary condition for optimality results from requiring the Fréchet derivative of $\bar{J}$ evaluated along perturbation direction $\eta$ to vanish for all $\eta$, i.e.,

$$
\left.\frac{d}{d \epsilon} \bar{J}\left(\delta x_{0}+\epsilon \eta\right)\right|_{\epsilon=0}=0 \quad \forall \eta .
$$

Since $P_{0}^{-1}$ and $R^{-1}$ are symmetric matrices, this yields

$$
\eta^{T}\left(-\int_{t_{0}}^{t} \Phi\left(\tau, t_{0}\right)^{T} C^{T} R^{-1}\left(\Delta y-C \Phi\left(\tau, t_{0}\right) \delta x_{0}\right) d \tau+P_{0}^{-1} \delta x_{0}\right)=0 .
$$

Since this expression is zero for any $\eta$, the parenthesized terms must be identically zero, yielding the linear equation for $\delta x_{0}$

$$
\left(\int_{t_{0}}^{t} \Phi^{T} C^{T} R^{-1} C \Phi d \tau+P_{0}^{-1}\right) \delta x_{0}=\int_{t_{0}}^{t} \Phi^{T} C^{T} R^{-1} \Delta y d \tau
$$

${ }^{2}$ The term smooth does not refer to differentiablility here. A smoothing algorithm uses measurements over the entire estimation time interval simulataneously during each estimation step. where $\Phi=\Phi\left(\tau, t_{0}\right)$. Note the left-hand-side of (21) contains both the linear stochastic observability Gramian $\mathcal{W}_{\text {so }}\left(t_{0}, t\right)$ and the background error covariance. This equation may be solved uniquely for $\delta x_{0}$ if and only if the matrix inverse of the parenthesized term in (21) exists, i.e., it is full rank. Typically, the observability Gramian should be full rank to able to infer the initial state of the system. Here this requirement is relaxed because the presence of $P_{0}^{-1}$ ensures invertibility. In 4D-Var data assimilation, the parenthesized term is known as the reduced Hessian [20]. Due to the formulation in terms of observability with the addition of prior inverse covariance $P_{0}$, we refer to this matrix as the augmented observability Gramian

$$
\mathcal{W}_{\mathrm{ao}}\left(t_{0}, t\right)=\mathcal{W}_{\mathrm{so}}\left(t_{0}, t\right)+P_{0}^{-1} .
$$

For linear, discrete-time dynamics, Li and Navon [20] have shown the equivalence of 4D-Var and Kalman filter estimates. They have also shown a connection between the inverse of the reduced Hessian and the covariance of the Kalman Filter in discrete-time. These results motivate the solution that we now present of the covariance of the Kalman filter for deterministic dynamics in continuous time.

\subsection{Covariance for the Continuous-time Kalman Filter under Deterministic Dynamics with Initial Covariance $P_{0}$}

The solution (14) to (13) (equivalently (12) with $Q(t) \equiv 0$ ) requires the assumption of zero prior information. The solution to (12) when prior information is present was noted to exist [21] in the work of Levin [22]. However, the solution in [22] requires knowledge of all entries of a state transition matrix for an associated Hamiltonian dynamical system (which is not easily found, even in the time-invariant case), and the solution is not given explicitly in terms of the stochastic observability Gramian. We now present a solution for the inverse covariance of the continuous-time Kalman Filter for deterministic dynamics. To the best of our knowledge, it is the first solution to (13) in the presence of a background error covariance expressed in terms of the stochastic observability Gramian.

Since augmented observability $\mathcal{W}_{\mathrm{ao}}\left(t_{0}, t\right)$ is a quantity that results from a smoothing operation (4D-Var) and applies at time $t_{0}$, and (13) is a filtering equation applicable at time $t$, to solve (13) the augmented observability $\mathcal{W}_{\text {ao }}\left(t_{0}, t\right)$ must be transformed by $\Phi^{T}\left(t_{0}, t\right)$ and $\Phi\left(t_{0}, t\right)$ similar to (15).

Proposition 1. The inverse covariance of the continuous-time Kalman filter under deterministic linear dynamics and in the presence of initial covariance $P_{0}$ is

$$
\begin{aligned}
P^{-1}(t) & =\Phi^{T}\left(t_{0}, t\right) \mathcal{W}_{a o}\left(t_{0}, t\right) \Phi\left(t_{0}, t\right) \\
& =\Phi^{T}\left(t_{0}, t\right)\left(\mathcal{W}_{s o}\left(t_{0}, t\right)+P_{0}^{-1}\right) \Phi\left(t_{0}, t\right) .
\end{aligned}
$$

Proof. Note at $t=t_{0}, \Phi\left(t_{0}, t_{0}\right)=\mathbb{I}$ and $\mathcal{W}_{\text {so }}\left(t_{0}, t_{0}\right)=0$, hence $P^{-1}\left(t_{0}\right)=P_{0}^{-1}$ as desired. We must verify that $(23)$ is a solution to (13). Consider the left-hand-side of (13) by differentiating the proposed solution, which gives

$\frac{d}{d t}\left(P^{-1}(t)\right)=\dot{\Phi}^{T}\left(\mathcal{W}_{\mathrm{so}}+P_{0}^{-1}\right) \Phi+\Phi^{T} \frac{d}{d t}\left(\mathcal{W}_{\mathrm{so}}\right) \Phi+\Phi^{T}\left(\mathcal{W}_{\mathrm{so}}+P_{0}^{-1}\right) \dot{\Phi}$ 
where $\Phi=\Phi\left(t_{0}, t\right)$ and $\mathcal{W}_{\text {so }}=\mathcal{W}_{\text {so }}\left(t_{0}, t\right)$. Differentiation of $\mathcal{W}_{\text {so }}\left(t_{0}, t\right)$ yields

$$
\frac{d}{d t}\left(W_{\mathrm{so}}\left(t_{0}, t\right)\right)=\Phi^{T}\left(t, t_{0}\right) C^{T}(t) R^{-1}(t) C(t) \Phi\left(t, t_{0}\right) .
$$

Recall the transition matrix property $\dot{\Phi}\left(t_{0}, t\right)=-\Phi\left(t_{0}, t\right) A(t)$. Substituting (25) into (24) and using the composition property to show $\Phi^{T}\left(t_{0}, t\right) \Phi^{T}\left(t, t_{0}\right)=\mathbb{I}$ and $\Phi\left(t, t_{0}\right) \Phi\left(t_{0}, t\right)=\mathbb{I}$ results in the desired left-hand-side. Plugging (23) into the right-hand side of (13) gives a matching expression.

This result is important because it connects the optimal filter covariance to the augmented observability and enables the following distinctions between path-planning methods.

\subsection{Distinctions of path-planning strategies}

The optimal inverse covariance (23) is a transformed version of the augmented observability. Alternately, write (23) by factoring out an inverse, i.e.,

$$
P(t)=\Phi\left(t, t_{0}\right) W_{\text {ao }}^{-1}\left(t_{0}, t\right) \Phi^{T}\left(t, t_{0}\right) .
$$

Since a covariance matrix $P$ transforms under dynamics from $t_{1}$ to $t_{2}$ according to $P\left(t_{2}\right)=\Phi\left(t_{2}, t_{1}\right) P\left(t_{1}\right) \Phi^{T}\left(t_{2}, t_{1}\right)$ [16], the filtering covariance $P(t)$ at time $t$ is related to the inverse of the augmented observability $\mathcal{W}_{\text {ao }}^{-1}\left(t_{0}, t\right)$ by propagation under the dynamics. Equation (26) highlights the differences between path planning strategies in the linear case. Planning based on $\mathcal{W}_{\text {so }}$ alone does not account for prior covariance $P_{0}$. Planning based on the augmented observability Gramian $\mathcal{W}_{\text {ao }}$ accounts for both $\mathcal{W}_{\text {so }}$ and $P_{0}$, but still differs from filtering-covariance planning by the additional transformation of the dynamics.

These distinctions lead to the following interpretations of the strategies: (i) planning based on $\mathcal{W}_{\text {so }}$ is equivalent to selecting the candidate path that results in highest observability for the time interval $\left[t_{0}, t\right]$; (ii) planning based on $P(t)$ selects the candidate path that results in the most certainty in the antipicated posterior covariance at time $t$; (iii) planning based on $\mathcal{W}_{\text {ao }}$ selects the candidate path with observability that is the most complementary to information in the prior inverse covariance $P_{0}^{-1}$ at time $t_{0}$. Planning based on $\mathcal{W}_{\text {ao }}$ and $P(t)$ are not guaranteed to yield the same results since the transformation by the dynamics in (26) may affect the eigenvalues of the $\mathcal{W}_{\text {ao }}$. However, three practical advantages of planning with $\mathcal{W}_{\text {ao }}$ instead of $P(t)$ are (i) since the true state and flow-field parameters are not known for a flow estimation application, propagation of $\mathcal{W}_{\text {ao }}$ may not lead to a reliable anticipated posterior covariance; (ii) for nonlinear dynamics, the anticipated posterior probability density function may be multimodal and not well represented by $P(t)$; and (iii) the influence on path selection of the prior information encoded within $P_{0}$ may be easier to interpret since $P_{0}$ does not receive additional transformation.

\section{Empirical Augmented Observability-Based Path Planning}

\subsection{Empirical Augmented Observability}

To extend the linear augmented observability Gramian $\mathcal{W}_{\text {ao }}$ in (22) to nonlinear dynamics, we replace the linear stochastic observability Gramian $\mathcal{W}_{\text {so }}\left(t_{0}, t\right)$ with the stochastic version of the empirical observability Gramian $\mathcal{W}_{\mathrm{eo}}\left(t_{0}, t\right)$ defined in (5). The empirical augmented observability Gramian is

$$
\begin{aligned}
\mathcal{W}_{\mathrm{ea}}\left(t_{0}, t\right) & =\int_{t_{0}}^{t} \Psi_{\mathrm{e}}\left(\tau, t_{0}, x_{0}\right)^{T} R(\tau)^{-1} \Psi_{\mathrm{e}}\left(\tau, t_{0}, x_{0}\right) d \tau+P_{0}^{-1} \\
& =\mathcal{W}_{\mathrm{eo}}\left(t_{0}, t\right)+P_{0}^{-1}
\end{aligned}
$$

We also define the associated path-planning score to be the augmented unobservability index

$$
v_{a}\left(\mathcal{W}_{\mathrm{ea}}\right)=\frac{1}{\lambda_{\min }\left(\mathcal{W}_{e a}\right)}=\frac{1}{\lambda_{\min }\left(\mathcal{W}_{e o}+P_{0}^{-1}\right)} .
$$

For two matrices $V$ and $W$, it can be shown through use of an eigenvector of $V+W$ and Rayleigh quotients that $\lambda_{\min }(V)+$ $\lambda_{\min }(W) \leq \lambda_{\min }(V+W)$ [23]. This leads to the upper bound

$$
v_{a}\left(\mathcal{W}_{\mathrm{ea}}\right) \leq \frac{v\left(\mathcal{W}_{\mathrm{eo}}\right)}{1+v\left(\mathcal{W}_{\mathrm{eo}}\right) / \lambda_{\max }\left(P_{0}\right)},
$$

where we have used the fact that $\lambda_{\min }\left(P_{0}^{-1}\right)=1 / \lambda_{\max }\left(P_{0}\right)$. Note that if we have perfect initial knowledge of the state so that $P_{0} \rightarrow 0$, then the $v_{a}$ index tends to zero, indicating that the system is perfectly observable based on prior information alone. Similarly, if $v \rightarrow 0$, then $v_{a}$ goes to zero, so that perfect observability in $\mathcal{W}_{\text {eo }}$ leads to perfect observability in $\mathcal{W}_{\text {ea }}$. Also note that if we have no initial knowledge of the system state, i.e., $P_{0} \rightarrow \infty$, then $v_{a} \leq v$.

\subsection{Numerical Experiments with $\mathcal{W}_{\text {ea }}$}

Consider again the two-vortex system and the routes shown in Fig. 1(c). Route A reflected about both the real and imaginary axes yields Route B. Over the time interval $\left[T_{h}, 2 T_{h}\right]$, Routes A and $\mathrm{B}$ yield identical $v$ indices of 8.444 , whereas Route $\mathrm{C}$ has a $v$ index of 27.50. Now consider the $3 \times 3$ block submatrices in $\mathcal{W}_{\text {eo }}\left(T_{h}, 2 T_{h}\right)$ corresponding to the vortex states. Table 2 provides the $v$ indices for the vortex submatrices for each route. Route A and Route B yield identical yet interchanged values.

Table 2: Unobservability analysis of vortex states only for Routes A-C
\begin{tabular}{c|cc}
\multicolumn{3}{|c}{ Time span: $\left[T_{h}, 2 T_{h}\right]$} \\
\hline Route & Unobs., Vortex 1 & Unobs., Vortex 2 \\
\hline A & 0.2631 & 0.5933 \\
B & 0.5933 & 0.2631 \\
C & 0.1430 & 2.6468 \\
\hline
\end{tabular}

Traversing Route A around Vortex 1 provides more observability of Vortex 1 than Vortex 2, and traversing Route B around Vortex 2 provides more observability of Vortex 2 than Vortex 1. Route $\mathrm{C}$ is more complex, because it provides more observability of Vortex 1 even though it departs from Vortex 1 and begins to encircle Vortex 2.

The following numerical experiment illustrates the use of the empirical augmented observability for path planning. At time $t=T_{h}$, assume the vehicle has no prior observability information, but has a prior covariance $P_{0}=\operatorname{diag}\left(\alpha \mathbb{I}_{3 \times 3}, \beta \mathbb{I}_{3 \times 3}\right.$, $\left.0.001 \mathbb{I}_{2 \times 2}\right)$. That is, the vehicle knows its position with relative 

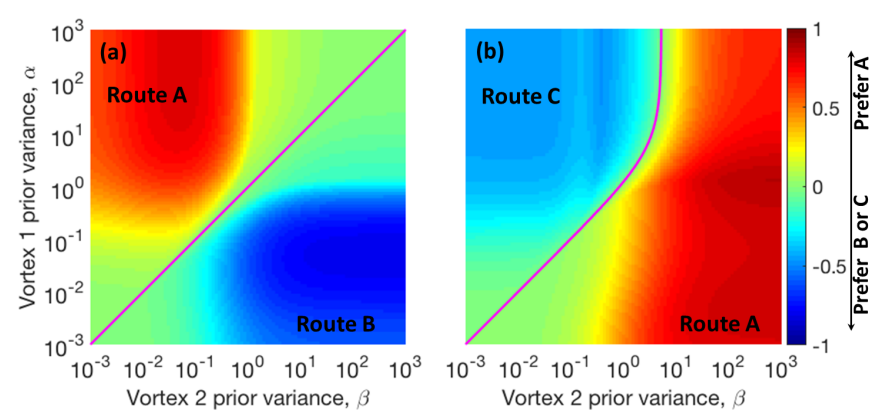

Figure 2: Turn confidence in selecting either (a) Route A or Route B, or (b) Route A or Route C, for a $100 \times 100$ grid of $\alpha$ and $\beta$ values. Decision boundaries where $v_{a, j}=v_{a, A}$ are shown as magenta lines.

certainty and it has $\alpha, \beta \in \mathbb{R}^{+}$multipliers for diagonal covariances on Vortices 1 and 2, respectively. If $\alpha>\beta$, then the vehicle has more initial uncertainty about Vortex 1 as compared to Vortex 2, and vice versa. For the planning interval $\left[T_{h}, 2 T_{h}\right]$, the vehicle chooses a route using the augmented observability index $v_{a}$. For ease of interpretation of the results, first consider the case in which the vehicle may impulsively switch to Route B. Define the quantity $\left(v_{a, j}-v_{a, A}\right) / \max \left(v_{a, A}, v_{a, j}\right)$ for Routes $j=B, C$ as a measure of turn confidence. When $v_{a, j}>v_{a, A}$, turn confidence is positive and the vehicle should remain on Route A. When $v_{a, j}<v_{a, A}$, the vehicle should switch to Route $j$ for $j=B, C$. The denominator maps to the interval $[-1,1]$.

Figures 2(a) and 2(b) show the results of numerically altering the $\alpha$ and $\beta$ prior variances. For large, uncertain $\alpha$ or $\beta$ values, the prior covariance term determines the turn selection in Fig. 2(a), and the vehicle appropriately selects the route that explores the more uncertain vortex. In Fig. 2(b), the behavior is more complex due to the observability results associated with this route in Table 2 . Route $\mathrm{C}$ provides more observability of Vortex 1 relative to Vortex 2, and it also provides more observability of Vortex 1 than Route A does. Route A provides more observability of Vortex 2 than Route C. Correspondingly, turn selections in Fig. 2(b) have reversed. Also note that Fig. 2(b) displays a region where the diagonal decision boundary bends. In this region, $\alpha$ and $\beta$ are large and uncertain, so the vehicle's decision defaults to the observability analysis without prior information in Table 1; Route A is selected because it is more observable than Route $\mathrm{C}$. These experiments suggest that the augmented unobservability index yields a criterion for automated path selection that coincides with intuitive use of observability in a manner that complements the prior information of the background error covariance $P_{0}$.

\section{Conclusion}

This paper addresses automatic route selection in path planning for flow estimation by scoring candidate trajectories using a new measure called the augmented unobservability index. This measure includes a background error covariance as well as a forward-looking observability analysis. We define augmented observability by drawing insight from the continuoustime reduced Hessian in 4D-Var data assimilation. We compare augmented observability to the optimal, anticipated in- verse covariance for a linear, deterministic continuous-time system with measurement noise, and we highlight distinctions between path-planning strategies. A numerical experiment shows the benefit of augmented empirical observability for turn selection in navigating a two-vortex flow field. The appendix also connects empirical augmented observability to information theory. In ongoing work, we are applying this novel path-planning strategy for closed-loop estimation of a two-vortex flow field.

\section{Acknowledgments}

FDL acknowledges helpful discussions with P. S. Krishnaprasad and Yunlong Huang. This research received support from National Science Foundation Award No. CMMI-1362837.

\section{References}

[1] A. Alvarez, B. Garau, A. Caiti, Combining networks of drifting profiling floats and gliders for adaptive sampling of the ocean, in: Robot. Autom. 2007 IEEE Int. Conf., 2007, pp. 157-162.

[2] C. J. Cannell, D. J. Stilwell, A comparison of two approaches for adaptive sampling of environmental processes using autonomous underwater vehicles, Proc. MTS/IEEE Oceans Conf. (2005) 1514-1521 Vol. 2.

[3] N. E. Leonard, D. A. Paley, F. Lekien, R. Sepulchre, D. M. Fratantoni, R. E. Davis, Collective motion, sensor networks, and ocean sampling, Proc. IEEE 95 (2007) 48-74.

[4] S. Ramp, R. Davis, N. Leonard, I. Shulman, Y. Chao, A. Robinson, J. Marsden, P. Lermusiaux, D. Fratantoni, J. Paduan, F. Chavez, F. Bahr, S. Liang, W. Leslie, Z. Li, Preparing to predict: The Second Autonomous Ocean Sampling Network (AOSN-II) experiment in the Monterey Bay, Deep Sea Res. Part II Top. Stud. Oceanogr. 56 (2009) 68-86.

[5] B. T. Hinson, M. K. Binder, K. A. Morgansen, Path planning to optimize observability in a planar uniform flow field, in: 2013 Amer. Control Conf., Washington, DC, 2013, pp. 1394-1401.

[6] L. DeVries, S. J. Majumdar, D. A. Paley, Observability-based optimization of coordinated sampling trajectories for recursive estimation of a strong, spatially varying flowfield, J. Intell. Robot. Syst. 70 (2012) 527544.

[7] J. D. Quenzer, K. A. Morgansen, Observability based control in rangeonly underwater vehicle localization, Amer. Control Conf. (2014) 47024707.

[8] N. E. Leonard, D. A. Paley, R. E. Davis, D. M. Fratantoni, F. Lekien, F. Zhang, Coordinated control of an underwater glider fleet in an adaptive ocean sampling field experiment in Monterey Bay, J. Field Robotics 27 (2010) 718-740.

[9] F. D. Lagor, K. Ide, D. A. Paley, Touring invariant set boundaries of a two-vortex system using streamline control, in: 54th IEEE Conf. Decis. Control, Osaka, JP, 2015.

[10] F. D. Lagor, A. Davis, K. Ide, D. A. Paley, Non-Gaussian estimation of a two-vortex flow using a Lagrangian sensor guided by output feedback control, in: 2016 Amer. Control Conf., Boston, MA, 2016.

[11] C. H. Bishop, B. J. Etherton, S. J. Majumdar, Adaptive sampling with the ensemble transform Kalman filter. Part I: Theoretical aspects, Mon. Weather Rev. 129 (2001) 420-436.

[12] R. E. Davis, N. E. Leonard, D. M. Fratantoni, Routing strategies for underwater gliders, Deep Sea Res. Part II Top. Stud. Oceanogr. 56 (2009) $173-187$.

[13] A. J. Krener, K. Ide, Measures of unobservability, in: 48th IEEE Conf. Decis. Control, 2009, pp. 6401-6406.

[14] R. Brockett, Finite dimensional linear systems, John Wiley \& Sons, New York, 1970.

[15] N. D. Powel, K. A. Morgansen, Empirical observability Gramian rank condition for weak observability of nonlinear systems with control, in: 54th IEEE Conf. Decis. Control, Osaka, JP, 2015, pp. 6342-6348.

[16] A. Gelb, Applied optimal estimation, M.I.T. Press, Cambridge MA, 1974.

[17] M. Aoki, On observability of stochastic discrete-time dynamic systems, J. Franklin Inst. 286 (1968) 36-58. 
[18] F.-X. Le Dimet, O. Talagrand, Variational algorithms for analysis and assimilation of meteorological observations: Theoretical aspects, Tellus A 38A (1986) 97-110.

[19] P. Courtier, J. N. Thepaut, A. Hollingsworth, A strategy for operational implementation of 4D-Var, using an incremental approach, Q. J. R. Meteorol. Soc. 120 (1994) 1367-1387.

[20] Z. Li, I. Navon, Optimality of variational data assimilation and its relationship with the Kalman filter and smoother, Q. J. R. Meteorol. Soc. 127 (2001) 661-683.

[21] R. E. Kalman, R. S. Bucy, New results in linear filtering and prediction theory, J. Basic Eng. 83 (1961) 95-108.

[22] J. J. Levin, On the matrix Riccati equation, Proc. Amer. Math. Soc. 10 (1959) 519-524.

[23] G. Strang, Linear algebra and its applications, 4th Edition, Thomson Brooks/Cole, Belmont CA, 2006.

[24] S. Kay, Fundamentals of statistical signal processing, vol. I: estimation theory, Prentice Hall, Upper Saddle River N.J., 1993.

[25] K. Law, A. Stuart, K. Zygalakis, Data assimilation: A mathematical introduction, Springer International, Cham, 2015.

[26] B. Øksendal, Stochastic differential equations, 5th Edition, SpringerVerlag, Heidelberg, 2000.

[27] P. Maybeck, Stochastic models, estimation, and control: vol. I, Academic Press, New York, 1979.

\section{Appendix A. Connections to Fisher Information}

Let $p(Y \mid \Omega)$ denote the conditional probability density (pdf) associated with measurements $Y$ of the output of a system with an underlying parameter vector $\Omega$. Let $\mathbb{E}[\cdot]$ denote the expectation operator. From information theory, the Fisher information matrix (FIM), defined component-wise by [24]

$$
[\mathcal{F}(Y, \Omega)]_{j k}=-\mathbb{E}\left(\frac{\partial^{2}}{\partial \Omega_{j} \partial \Omega_{k}} \log p(Y \mid \Omega)\right),
$$

assesses the informativeness of measurements $Y$ in the inference of $\Omega$. The FIM inverse provides the Cramér-Rao lower bound on the covariance $C_{\hat{\Omega}}$ of estimates $\hat{\Omega}$ generated by an unbiased estimator (i.e., $C_{\hat{\Omega}} \geq F^{-1}$ ) [24].

One may distinguish versions of the FIM by the pdf substituted for $p(Y \mid \Omega)$. Linear information filters, which are Kalman filters formulated to propagate the inverse of the state covariance, use the filtering density $p(y(t) \mid x)$ for the measurement vector $y(t)$ conditioned on the signal $x(\cdot)$ over the interval $\left[t_{0}, t\right]$ to obtain $\mathcal{F}(y(t), x)=P^{-1}(t)$ given in (14). Powel and Morgansen [15] connect the time-derivative of the empirical observability Gramian to $F\left(y(t) \mid x\left(t_{0}\right)\right)$, which is based on the latest measurement and the initial condition, in the limit of (6) as $\epsilon \rightarrow 0$. Here, we consider the likelihood density $p\left(y \mid x\left(t_{0}\right)\right)$ for the continuous-time signal $y(\cdot)$ over the interval $\left[t_{0}, t\right]$, conditioned on the uncertain initial condition $x\left(t_{0}\right)$.

Calculation of the FIM using the $p\left(y \mid x\left(t_{0}\right)\right)$ density for signal $y(\cdot)$ requires stochastic analysis that properly accounts for the nonconstant offset $h(t, x(t))$ in the output equation of (3); we follow Law et al. [25], who derive a related expression for a posterior density. We consider a constant measurement covariance $R(t)=R$. Define the integrated stochastic process $Y(t)=\int_{t_{0}}^{t} y(\tau) d \tau$ and let $\beta \in \mathbb{R}^{m}$ be a standard Brownian motion with time shift $t_{0}$ such that $\beta\left(t_{0}\right)=0$ and $\mathbb{E}\left(\beta\left(t_{0}+1\right)^{2}\right)=1$. For a given $x\left(t_{0}\right)$ and the corresponding trajectory $\phi\left(\cdot, t_{0}, x\left(t_{0}\right)\right)$, the output equation of (3) can be represented by the Itô process [25]

$$
d Y(t)=h\left(t, \phi\left(t, t_{0}, x\left(t_{0}\right)\right)\right) d t+\sqrt{R} d \beta(t) .
$$

Let $Y_{R}(t)$ be an associated reference process that is the solution to the driftless version of (A.2), i.e., when $h\left(t, \phi\left(t, t_{0}, x\left(t_{0}\right)\right)\right)$ $\equiv 0$. For brevity, we suppress the arguments of $\phi$. For (A.2) with uncertain $x\left(t_{0}\right)$, Girsanov's formula [25], [26]

$$
\frac{p\left(Y, x\left(t_{0}\right)\right)}{p\left(Y_{R}, x\left(t_{0}\right)\right)}=\exp \left(-\frac{1}{2} \int_{t_{0}}^{t}\|h(\tau, \phi)\|_{R}^{2} d \tau+\int_{t_{0}}^{t}\langle h(\tau, \phi), d Y(\tau)\rangle_{R}\right)
$$

provides a means of obtaining the relevant joint density $p\left(Y, x\left(t_{0}\right)\right)$ in relation to a driftless reference system. The second integral in (A.3) is an Itô integral, where the bracketed integrand is the weighted inner product $\langle a, b\rangle_{R}=\left(R^{-1 / 2} a\right)^{T}\left(R^{-1 / 2} b\right)$. Girsanov's formula enables the following connection between empirical observability and the FIM.

Proposition 2. For system (3) with an uncertain initial condition $x\left(t_{0}\right) \sim \mathcal{N}\left(x_{0}, P_{0}\right)$ and measurement noise $v(t) \sim \mathcal{N}(0, R)$, the empirical observability Gramian (5) is a numerical approximation to the Fisher information matrix $\mathcal{F}\left(y, x\left(t_{0}\right)\right)$ if the likelihood density $p\left(y \mid x\left(t_{0}\right)\right)$ is differentially smooth.

Proof. The independence of $Y_{R}$ and $x\left(t_{0}\right)$ implies

$$
\frac{p\left(Y, x\left(t_{0}\right)\right)}{p\left(Y_{R}, x\left(t_{0}\right)\right)}=\frac{p\left(Y \mid x\left(t_{0}\right)\right)}{p\left(Y_{R}\right)} \text {. }
$$

By the property of $\log$ arithms $\log (A / B)=\log A-\log B$ and the absence of $x\left(t_{0}\right)$ from $p\left(Y_{R}\right)$, note that

$$
\frac{\partial^{2}}{\partial x\left(t_{0}\right)_{j} \partial x\left(t_{0}\right)_{k}} \log p\left(Y \mid x\left(t_{0}\right)\right)=\frac{\partial^{2}}{\partial x\left(t_{0}\right)_{j} \partial x\left(t_{0}\right)_{k}} \log \frac{p\left(Y \mid x\left(t_{0}\right)\right)}{p\left(Y_{R}\right)} .
$$

Combining (A.5) with (A.1) yields for $\left[\mathcal{F}\left(Y, x\left(t_{0}\right)\right)\right]_{j k}$

$$
\mathbb{E}\left(\frac{\partial^{2}}{\partial x\left(t_{0}\right)_{j} \partial x\left(t_{0}\right)_{k}}\left(\frac{1}{2} \int_{t_{0}}^{t}\|h(\tau, \phi)\|_{R}^{2} d \tau-\int_{t_{0}}^{t}\langle h(\tau, \phi), d Y(\tau)\rangle_{R}\right)\right) .
$$

By the smoothness assumption on $p\left(y \mid x\left(t_{0}\right)\right)$, the expectation and the partial differentiation operations may commute. The expectation of an Itô integral is zero [25], so the second term provides zero contribution. Differentiation of the first term yields

$$
\mathcal{F}\left(y, x\left(t_{0}\right)\right)=\int_{t_{0}}^{t} \frac{\partial h(\tau, \phi)^{T}}{\partial x\left(t_{0}\right)} R^{-1} \frac{\partial h(\tau, \phi)}{\partial x\left(t_{0}\right)} d \tau .
$$

Following [15] in passing (3) to a limit as $\epsilon \rightarrow 0$ gives

$$
\lim _{\epsilon \rightarrow 0} \mathcal{W}_{\mathrm{eo}}\left(t_{0}, t\right)=\mathcal{F}\left(y, x\left(t_{0}\right)\right) .
$$

This expression reveals that $\mathcal{W}_{\mathrm{eo}}\left(t_{0}, t\right)$ is an approximation to $\mathcal{F}\left(y, x\left(t_{0}\right)\right)$ for nonvanishing values of $\epsilon$.

The FIM assesses the inference certainty due to measurement data alone [27], so prior information does not appear in (A.8). Including prior information, the relation between the FIM and $\mathcal{W}_{\text {ea }}$ becomes

$$
\lim _{\epsilon \rightarrow 0} \mathcal{W}_{\mathrm{ea}}\left(t_{0}, t\right)=\mathcal{F}\left(y, x\left(t_{0}\right)\right)+P_{0}^{-1} .
$$

